[2] Nanki T, et al., Arthritis Rheum (2002) 46, 2878-83

[3] Hoshino K, et al., Arthritis Rheumatol (2018) Aug 6 [Epub ahead of print]

Disclosure of Interests: NAOTO ISHII Shareholder of: Eisai Co., Ltd., Employee of: KAN Research Institute, Inc. (subsidiary of Eisai Co., Ltd.), Kana Hoshino-Negishi Shareholder of: Eisai Co., Ltd., Employee of: KAN Research Institute, Inc. (subsidiary of Eisai Co., Ltd.), Masayoshi Ohkuro Employee of: EA Pharma Co., Ltd., Tomoya Nakatani Employee of: KAN Research Institute, Inc. (subsidiary of Eisai Co., Ltd.), Wataru Ikeda Shareholder of: Eisai Co., Ltd., Employee of: KAN Research Institute, Inc. (subsidiary of Eisai Co., Ltd.), Yoshikazu Kuboi Shareholder of: Eisai Co., Ltd., Employee of: KAN Research Institute, Inc. (subsidiary of Eisai Co., Ltd.), Nobuyuki Yasuda Shareholder of: Eisai Co., Ltd., Employee of: KAN Research Institute, Inc. (subsidiary of Eisai Co., Ltd.), Toshio Imai Shareholder of: Eisai Co., Ltd., Employee of: KAN Research Institute, Inc. (subsidiary of Eisai Co., Ltd.)

DOI: 10.1136/annrheumdis-2019-eular.1790

\section{SAT0049 INFECTION WITH CITRULLINATING PORPHYROMONAS GINGIVALIS CAN INDUCE AUTOIMMUNITY TO HUMAN RIBOSOMAL PROTEINS AND TNF ALPHA TREATMENT NONRESPONSE}

Madeleine Jenning ${ }^{1}$, Bianka Marklein ${ }^{1}$, Ute Nonhoff $^{2}$, Zoltan Konthur ${ }^{2}$, Gerd Rüdiger Burmester ${ }^{1}$, Karl Skriner ${ }^{1} .{ }^{1}$ Charité University Medicine, Department of Rheumatology and Clinical Immunology, Berlin, Germany, ${ }^{2}$ Max Plank Institute, Max-Planck-Institut für Kolloid- und Grenzflächenforschung, Potsdam, Germany

Background: Porphyromonas gingivalis ( $\mathrm{P}$ g.) is involved in triggering self-reactive immune responses when cirtullinating bacterial or human proteins. However, first evidence to link anti-ribosomal $T$ and $B$ cells responses to rheumatoid arthritis (RA) has been published but the mechanism and its influence on therapy is not clear (1). Infection based autoimmunity induced by citrullination of human proteins with $P$ g. peptidyl arginine deiminase from RA patient (RA-PPAD) and crossreactivity binding induced by $\mathrm{P} g$. was investigated using patient sera, affinity purified RA patient antibodies and monoclonal antibodies to cit-RA-PPAD.

Objectives: Antibodies to RA-PPAD isolated from an RA patients (RAPPAD) was first time linked to target specific citrullinated ribosomal proteins and therapy.

Methods: Screening of RA sera was conducted on 37.830 unique human proteins on protein marcoarrays (http://www.engine-gmbh.de) with $30 \mathrm{RA}$ sera. The autoantibody response to 840 different proteins was recorded and bioinformatically evaluated. Protein arrays were citrullinated with human peptidyl arginine deiminase PAD 2, 4, rabbit PAD and RA-PPAD from P.g. Sera and affinity purified antibodies were used to detect reactivity to 840 autoantigens and 15aa CCP peptide form RA-PPAD. Sera from TBA treated sera anti-TNF (adalimumab, etanercept, certolizumab) treatment were tested with the cit-PPAD-peptide of 15aa (CPP).

Results: A human protein macroarray consisting of 840 indentified autoantigens from RA patients was modified by human PAD2 and PAD4, rabbit $P A D$, and RA-PPAD form $P$ g. Using cit specific monoclonal antibodies we identified the ribosomal proteins (RP), RPL18a, RPS27a, modified by PAD2, RPL18a and MRPS11 modifies by PAD4, and RPL7L1 modified by rabbit PAD specifically targeted. In addition 6 RA patient sera and 3 osteoarthritis $(\mathrm{OA})$ control sera were used to identify the citrullinated RAPPAD specific modified autoantigens not targeted when modified by human PAD2 or PAD4 or rabbit PAD. We identified the RA-PPAD citrullinated ribosomal Proteins RPL3, RPL21, RPS24, RPL9, RPL15, RPS24, RPS3a, MRPL28 specifically targeted by RA patients. This identifies ribosomal proteins as major specific RA-PPAD citrullination targets. Moreover, affinity purified antibodies bound to native and citrullinated RA-PPAD from 6 RA patient sera and 3 OA patient sera were tested for crossreactvity on citrullinated human proteinarray. Antibodies to citrullinated ribosomal proteins MRPS11, RPL21, RPS3a, RPL18a, RPS27a, MRPL28 were detected in the RA group but not in the OA control group. High antibody titre against the cit-PPAD-peptide of 15aa (CPP) derived from the autocitrullination site (R63) of RA-PPAD correlates with TNFo-inhibitor (TBA) non-response $(n=61)$. DMARD patients refractory to different treatment regimes $(n=61)$, receiving anti-TNF (adalimumab, etanercept, certolizumab), do not respond when maintaining high $\alpha$-CPP lgG level.

Conclusion: Failure of Porphyromonas gingivalis clearance in RA patients leads to infection induced enzymatic mimicry based autoreactivity targeting evolutionary conserved human ribosomal proteins. Autoimmunity to ubiquitous self-antigens may trigger localized tissue damage in RA.TBA nonresponse leads to the suggestion to clear Porphyromonas gingivalis infection before $\alpha$-TNF treatment.

\section{REFERENCE}

[1] :Ito, Y. et al. Detection of $T$ cell responses to a ubiquitous cellular protein in autoimmune disease. Science 346, 363-368 (2014)

Disclosure of Interests: Madeleine Jenning: None declared, Bianka Marklein: None declared, Ute Nonhoff: None declared, Zoltan Konthur: None declared, Gerd Rüdiger Burmester Consultant for: Roche, Sanofi-Genzyme, Speakers bureau: Roche, Sanofi-Genzyme, Karl Skriner: None declared DOI: 10.1136/annrheumdis-2019-eular.4653

\section{SAT0050 TNF-A INDUCES MITOPHAGY FORMATION IN RHEUMATOID ARTHRITIS SYNOVIAL FIBROBLAST AND INHIBITION OF MITOPHAGY AMELIORATES SYNOVITIS IN COLLAGEN ANTIBODY-INDUCED ARTHRITIS}

sang youn jung. Bundang CHA medical Center, Department of Rheumatology, Seongnam, Gyeonggi-do, Korea, Rep. of (South Korea)

Background: Rheumatoid arthritis (RA) is a systemic and chronic autoimmune disease that primarily targets synovial membranes. Mitophagy is a selective form of autophagy which removes damaged mitochondria. When mitochondria become damaged, PINK1 (PTEN-induced putative kinase 1) is accumulated on the outer membrane of damaged mitochondria and induces mitophagy. The study of mitophagy in lung fibrosis and parkinson's diseases has recently been described. However, the role of mitophagy in RA has not been reported yet.

Objectives: The aim of this study was to determine the roles of mitophagy in rheumatoid arthritis synovial fibroblast (RASF) and collagen antibody-induced arthritis (CAIA) mice model.

Methods: We studied the mitophagy marker, PINK1, in RASF treated with TNF-a by western blotting and immunofluorescence. Arthritis was induced in PINK1 knockout mice by intraperitoneal (i.p.) injection of antitype II collagen antibody cocktail, followed by i.p injection of lipopolysaccharide. The severity of rheumatoid arthritis was histopathologically assessed

Results: PINK1 expression and damaged mitochondria increased in RASF treated with TNF- $\alpha$. TNF- $\alpha$ produced Intracellular ROS in RASF and mitophagy was regulated by ROS. PINK1 knockdown RASF decreased migration and invasion function. Histopathological examination revealed that the paws from PINK1-knockout mice showed markedly reduced swelling and inflammation compared with those of wild type mice

Conclusion: Taken together, the results suggest that regulation of PINK1 expression in RA may represent potential therapeutic and diagnostic targets for RA
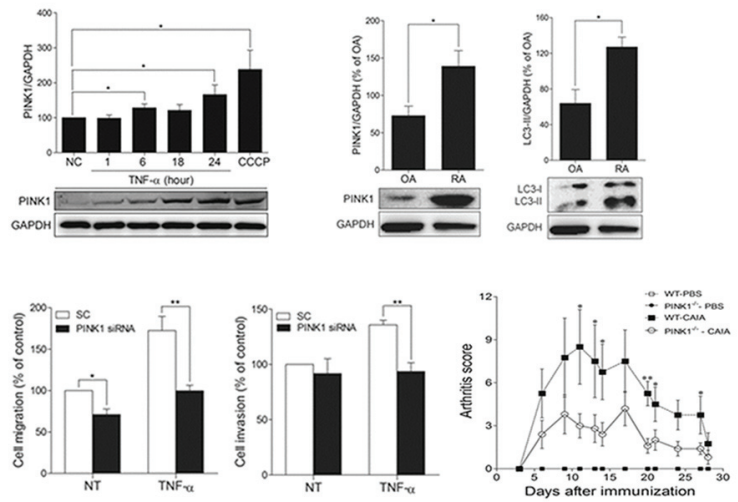

\section{REFERENCES}

[1] Huber LC, Distler O, Tarner I, Gay RE, Gay S, Pap T. Synovial fibroblasts: key players in rheumatoid arthritis. Rheumatology (Oxford). 2006;45 (6):669-75 